Research Article

\title{
Output-Feedback Sliding Mode Control for Permanent Magnet Synchronous Motor Servo System Subject to Unmatched Disturbances
}

\author{
Wei Jiang $\mathbb{i D}^{1}$ and Lu Zhang $\mathbb{D}^{2}$ \\ ${ }^{1}$ Department of Electronic Information, Wuxi Vocational Institute of Arts and Technology, Wuxi, China \\ ${ }^{2}$ School of Automation, Southeast University, Nanjing, China \\ Correspondence should be addressed to Wei Jiang; jiangwei@wxgyxy.cn
}

Received 14 November 2020; Revised 30 December 2020; Accepted 3 March 2021; Published 15 March 2021

Academic Editor: Richard I. Avery

Copyright (c) 2021 Wei Jiang and Lu Zhang. This is an open access article distributed under the Creative Commons Attribution License, which permits unrestricted use, distribution, and reproduction in any medium, provided the original work is properly cited.

\begin{abstract}
This paper aims to investigate the speed regulation problem for permanent magnet synchronous motor (PMSM) servo systems subject to unknown load torque disturbances. The proposed method utilizes sliding mode control (SMC), invariant manifold theory, and disturbance observation technique. In the PMSM servo systems, the unknown load torques will affect the control performance to a large extent, which is unmatched. In addition, compared with full-state measurement, the output-feedback framework is easy to implement and reduces the sensor costs. However, it is difficult to handle unmatched disturbance and unmeasured states simultaneously. To this end, this paper specifically combines the sliding mode control theory with the invariant manifold theory and puts forward an output-feedback disturbance rejection control method. The key idea is that the unmatched disturbance in the PMSM servo systems is transformed into matched one by taking advantage of the invariant manifold, which is different from existing results. The transformation maintains most of dynamics of the PMSM system for control design, which improves the accuracy. In addition, an extended state observer is designed to estimate the current and lumped disturbance simultaneously; then, the output-feedback SMC method is proposed by introducing the estimations. Besides, the switching gain in the proposed sliding mode controller can change with estimation errors adaptively, and the chattering reduces. Simulation results on a PMSM system validate the effectiveness of the proposed control strategy.
\end{abstract}

\section{Introduction}

Permanent magnet synchronous motor (PMSM) has been widely applied to various practical systems, such as robotics, aerospace, and power generations [1-4], due to the highefficiency, high air-gap flux density, large torque-to-inertia ratio, and high power density [1]. High-accuracy tracking performance and satisfactory dynamic response are significant in the aforementioned applications. However, it should be noted that the servo control performance is significantly affected by nonlinearities, uncertainties, and disturbances in PMSM systems, and the traditional linear control strategies including the proportional-integral (PI) controller [5] are unable to provide satisfactory control performance [6]. In order to obtain better performance, many advanced nonlinear control methods have been developed for PMSM servo systems in recent years, such as adaptive control $[2,7]$, robust control [8, 9], linearization control [10], disturbance observer-based control $[2,11]$, fuzzy-logic based control $[6,12]$, finite time control $[13,14]$, fractional order control [15], sliding mode control [16, 17], and neuro-network based control $[5,12]$. These control strategies improve control performance for PMSM servo systems from different aspects.

In industrial applications, sliding mode control (SMC) is deemed as one of the most effective control techniques $[18,19]$ due to its conceptual simplicity and powerful ability to reject matched disturbances/uncertainties [20]. The SMC is able to force the system dynamics to reach a predesigned sliding manifold in a finite time [21], and then the system 
trajectory tracking error will converge to zero along the sliding manifold $[22,23]$. It is well known that the load torque disturbance in PMSM system is unmatched one if we combine the speed control loop and current control loop together to get fast speed regulation performance [8]. In [24], a disturbance observer-based SMC strategy is developed to handle unmatched disturbances effectively. In [25], an adaptive sliding mode control method is proposed to attenuate the inductance disturbance of the PMSM. In order to estimate the unmeasured mechanical parameters of PMSM, a terminal sliding mode observer is proposed in [26], which can estimate the control performance with a finitetime convergence rate. The work in [27] employs the sliding mode control technique and the extended state observer for PMSM system to improve the robustness against load disturbance and parameter variations. These results are mostly implemented in the condition that all the system states are available. However, in PMSM servo systems, there are measurement noises in current sensors, which will bring adverse effects to the control performance. Toward that end, it is of great importance to investigate output-feedback SMC approaches for PMSM systems, which only require the interested output measurement rather than the full states.

The widely used output-feedback SMC method in PMSM is the observer-based SMC, which develops a state observer $[28,29]$ to reconstruct all the unmeasured states and then designs a controller by utilizing the estimation values. Most of the existing observer-based SMC methods need large control gains to reject bounded equivalent input disturbances [30], which will cause serious chattering. In recent years, the output-feedback SMC based upon the extended state observer (ESO) [31] provides an intuitive solution for chattering attenuation [32]. In [33], a higherorder ESO-based SMC method is proposed to reject the unknown higher-order lumped disturbances.

It is noticed that the existing ESO-based SMC method for PMSM system requires the second-order derivatives of the angular velocity to obtain a standard form for control design [33]. Under the standard ESO-based framework, the dynamics except for the integral chain are lumped for estimation. The lumped disturbances and uncertainties may change in a wide range due to the variation of operation condition. Therefore, to achieve satisfactory estimation and control performance, it is generally required to assign relatively large observer poles. For a PMSM servo system, the increase of observer poles will result in a drastic increase of observer gains, and the measurement noises will be significantly amplified in practice. Therefore, there will be serious switching action in the control signal, which will bring adverse effects to actuators and even excites the unmodelled dynamics of the system [34]. Besides, the derivatives of the reference signal may be unavailable. The ESO-based methods solve this problem by adding tracking differentiators for online estimation of the unknown information, which will considerably increase the complexity for implementation. Taking all the aforementioned facts into account, it is imperative to investigate some new outputfeedback SMC strategies for PMSM system subject to unmatched disturbances to address the above problems and to achieve good disturbance rejection ability and satisfactory control performance.

Actually, the output-feedback SMC for PMSM servo system subject to unknown load torque disturbances is a challenging problem, since it is difficult to estimate both the unmeasured states and the unmatched disturbance at the same time. Reference [35] gives a new idea for outputfeedback SMC design, which combines SMC with the basic idea of output regulation theory [36, 37]. Inspired by [35], this paper aims to solve the output-feedback SMC problem for PMSM servo system subject to unmatched disturbances. Under the proposed controller, the angular velocity is driven to track the reference signal asymptotically. The main contributions and benefits of the proposed method are summarized as follows: (1) full dynamics of the PMSM system are thoroughly exploited in the invariant manifold based output-feedback SMC design process, which admits higher bandwidth without higher observer gains, attenuating measurement noises to a large extent; (2) the switching gain changes with estimation errors adaptively, and thus the chattering will be reduced; (3) the proposed output-feedback SMC method can compensate the influences caused by unknown derivatives of the desired reference signal without resorting to tracking differentiators, which results in a simpler control structure and saves the implementation burden of the algorithm. To further demonstrate the effectiveness of the output-feedback SMC method proposed in this paper, simulations on a PMSM system are carried out by utilizing MATLAB/ Simulink.

The paper is organized as follows. Section 2 shows the mathematical model of the PMSM system and formulates the problem. In Section 3, the output-feedback sliding mode controller is developed and stability analysis is shown in detail. Then, Section 4 gives the simulation results and the conclusion is shown in Section 5.

\section{System Model and Problem Formulation}

2.1. Mathematical Model of PMSM. The magnetic circuit is assumed to be unsaturated, the eddy current loss and hysteresis are ignored, and the distribution of the magnetic field is supposed to be in sine space. A three-phase surfacemounted PMSM system can be modeled in $d-q$ coordinate, and the mathematical model is given by [2]

$$
\begin{aligned}
& \frac{\mathrm{d} i_{d}}{\mathrm{~d} t}=-\frac{R}{L} i_{d}+n_{p} \omega i_{q}+\frac{1}{L} u_{d}, \\
& \frac{\mathrm{d} i_{q}}{\mathrm{~d} t}=-n_{p} \omega i_{d}-\frac{R}{L} i_{q}-\frac{1}{L} n_{p} \omega \psi_{f}+\frac{1}{L} u_{q}, \\
& \frac{\mathrm{d} \omega}{\mathrm{d} t}=\frac{3 n_{p} \psi_{f}}{2 J} i_{q}-\frac{B}{J} \omega-\frac{T_{L}}{J},
\end{aligned}
$$

where $i_{q}$ and $i_{d}$ are the $q$-axis and $d$-axis stator currents, respectively; $u_{q}$ and $u_{d}$ are the $q$-axis and $d$-axis stator voltages, respectively; $\psi_{f}$ is the rotor flux linkage; $n_{p}$ is the number of pole pairs; $J$ is the rotor inertia; $L$ is the stator inductance; $R$ is the stator resistance; $B$ is the viscous friction 
coefficient; $\omega$ is the angular velocity; and $T_{L}$ is the unknown load torque.

The field-oriented control is one of the widely used control frameworks in PMSM, and Figure 1 shows the schematic diagram. Under this framework, the flux-producing and torque-producing components of the stator current are decoupled such that flux controls and the independent torque are possible as those in DC motors. In PMSM, the controllers take advantage of the cascade control loop, which includes two current loops and a speed loop. In the two current loops, two PI controllers are used. Here, we pay our attention to developing a controller for speed loop to reject the unknown load torque disturbance.

In a PMSM servo system, it is always expected to obtain maximum torque; thus, there should be $i_{d}=0$. Therefore, the mathematical model (1) can be decoupled as the following form:

$$
\begin{aligned}
& \frac{\mathrm{d} i_{q}}{\mathrm{~d} t}=-\frac{R}{L} i_{q}-\frac{1}{L} n_{p} \omega \psi_{f}+\frac{1}{L} u_{q}, \\
& \frac{\mathrm{d} \omega}{\mathrm{d} t}=\frac{3 n_{p} \psi_{f}}{2 J} i_{q}-\frac{B}{J} \omega-\frac{T_{L}}{J},
\end{aligned}
$$

where $u_{q}$ is the control signal and $T_{L}$ is the unknown unmatched disturbance. This paper mainly explores the control strategy for the decoupled system given by (2).

2.1.1. Control Objective. This paper attempts to utilize the sliding mode control theory to address speed regulation problem for PMSM servo systems subject to unmatched disturbances. For system (2), an output-feedback sliding mode controller combining invariant manifold with disturbance observer is designed to drive the angular velocity $\omega$ to track the reference signal $\omega^{*}$ asymptotically.

2.2. Traditional Output-Feedback SMC Design. Define the system output tracking error as $y=\omega-\omega^{*}$. Taking the second-order derivative of $y$, one obtains [33]

$$
\begin{aligned}
\dot{y}= & \dot{\omega}-\dot{\omega}^{*}=\frac{3 n_{p} \psi_{f}}{2 J} i_{q}-\frac{B}{J} \omega-\frac{T_{L}}{J}-\dot{\omega}^{*} \\
y= & \frac{3 n_{p} \psi_{f}}{2 J} \dot{i}_{q}-\frac{B}{J} \dot{\omega}-\frac{\dot{T}_{L}}{J}-\ddot{\omega}^{*} \\
= & -\left(\frac{3 n_{p} \psi_{f} R}{2 J L}+\frac{3 n_{p} \psi_{f} B}{2 J^{2}}\right) i_{q}-\left(\frac{3 n_{p}^{2} \psi_{f}^{2}}{2 J L}-\frac{B^{2}}{J^{2}}\right) \omega \\
& +\frac{B T_{L}}{J}-\frac{\dot{T}_{L}}{J}+\frac{3 n_{p} \psi_{f}}{2 J L} u_{q} .
\end{aligned}
$$

Denoting $\quad D=-\left(\left(3 n_{p} \psi_{f} R / 2 J L\right)+\left(3 n_{p} \psi_{f} B / 2 J^{2}\right)\right) i_{q}$ $-\left(\left(3 n_{p}^{2} \psi_{f}^{2} / 2 J L\right)-\left(B^{2} / J^{2}\right)\right) \omega+\left(B T_{L} / J\right)-\left(\dot{T}_{L} / J\right)-\ddot{\omega}^{*}$ as the lumped disturbances and $\kappa=\left(3 n_{p} \psi_{f} / 2 J L\right)$ as the control coefficient, one has the following input-output formulation:

$$
\ddot{y}=D+\kappa u_{q} .
$$

Denote $v_{1}=y, v_{2}=\dot{y}$, and $v_{3}=D$. Then we have the following controllable state-space model:

$$
\left[\begin{array}{l}
\dot{v}_{1} \\
\dot{v}_{2} \\
\dot{v}_{3}
\end{array}\right]=\left[\begin{array}{lll}
0 & 1 & 0 \\
0 & 0 & 1 \\
0 & 0 & 0
\end{array}\right]\left[\begin{array}{l}
v_{1} \\
v_{2} \\
v_{3}
\end{array}\right]+\left[\begin{array}{l}
0 \\
\kappa \\
0
\end{array}\right]+\left[\begin{array}{l}
0 \\
0 \\
\omega
\end{array}\right] .
$$

It is assumed that the lumped disturbance $D$ and its derivative $\omega$ are bounded and satisfy $\lim _{t \rightarrow \infty} \omega=0$. On the basis of (5), the observer is constructed as

$$
\begin{aligned}
& \dot{\hat{v}}_{1}=\widehat{v}_{2}+l_{1}\left(v_{1}-\widehat{v}_{1}\right), \\
& \dot{\hat{v}}_{2}=\widehat{v}_{3}+l_{2}\left(v_{1}-\widehat{v}_{1}\right)+\kappa u_{q}, \\
& \dot{\hat{v}}_{3}=l_{3}\left(v_{1}-\widehat{v}_{1}\right),
\end{aligned}
$$

where $l_{1}, l_{2}$, and $l_{3}$ are observer gains. Introducing the estimations, the sliding manifold is developed as

$$
s=\widehat{v}_{2}+\beta \widehat{v}_{1},
$$

where $\beta>0$. The output-feedback controller is

$$
u_{q}=-\frac{1}{\kappa}\left[\widehat{v}_{3}+\beta \widehat{v}_{2}+k_{1} \operatorname{sgn}(s)+k_{2} s\right] \text {, }
$$

where $k_{1}=\rho+\left|\left(\beta l_{1}+l_{2}\right)\left(\nu_{1}-\widehat{v}_{1}\right)\right|, \rho>0, k_{2}>0$.

2.3. Motivations. For the PMSM system (2) subject to unmatched disturbance, the effects caused by unmatched disturbance can be removed by the traditional outputfeedback sliding mode controller effectively. Nevertheless, it is seen from coordinate transformation given by (3) and (4) that all unmeasured states are lumped into $D$, which includes $q$-axis current and load torques. In order to obtain satisfactory performance, we have to choose large observer poles; thus, large observer gains are required. As a result, measurement noises are amplified, and high-frequency fluctuations will be brought in control signal, which may excite the unmodelled dynamics of PMSM system, cause adverse effects on actuator, and even destabilize the system.

\section{Main Results}

3.1. Output-Feedback Sliding Mode Controller Design. Motivated by the basic idea of output regulation theory $[36,38]$, the steady states of the PMSM system (2) are calculated from regulator equations, which are shown as follows:

$$
\begin{aligned}
\frac{\mathrm{d} \pi_{i_{q}}}{\mathrm{~d} t} & =-\frac{R}{L} \pi_{i_{q}}-\frac{1}{L} n_{p} \psi_{f} \pi_{\omega}+\frac{1}{L} \pi_{u_{q}}, \\
\frac{\mathrm{d} \pi_{\omega}}{\mathrm{d} t} & =\frac{3 n_{p} \psi_{f}}{2 J} \pi_{i_{q}}-\frac{B}{J} \pi_{\omega}-\frac{T_{L}}{J}, \\
0 & =\pi_{\omega}-\omega^{*},
\end{aligned}
$$

where $\pi_{i_{q}}, \pi_{\omega}$, and $\pi_{u_{q}}$ represent the steady states of the PMSM system states $i_{q}$, $\omega$, and the control input $u_{q}$, respectively. It is obvious that the solutions of (9) are 


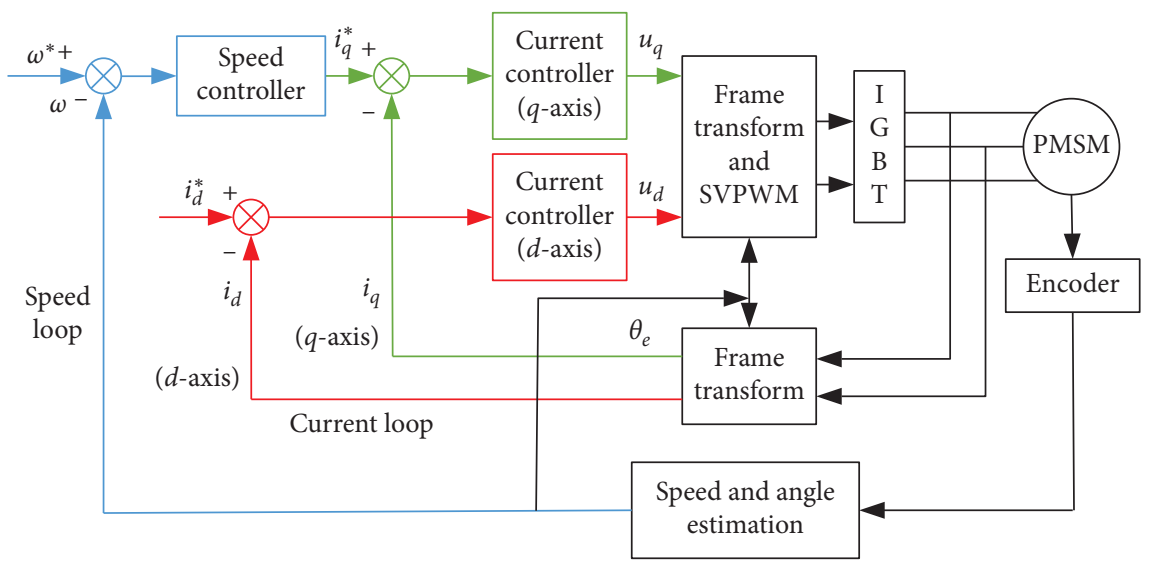

FIGURE 1: Schematic diagram of field-oriented control for PMSM.

impossible to be obtained due to the presence of unknown load torque disturbance $T_{L}$. This section will take advantage of the observation technique and sliding mode control to develop an output-feedback controller to solve this problem, which will only need the computable $\pi_{\omega}=\omega^{*}$.

Denote $\varepsilon_{1}=i_{q}-\pi_{i_{q}}$ and $\varepsilon_{2}=\omega-\pi_{\omega}$. Combining the PMSM system model (2) with the regulator equations given in (9), the invariant manifold is introduced as $\bar{E}=\left\{\varepsilon_{1}, \varepsilon_{2} \mid\left[\varepsilon_{1}, \varepsilon_{2}\right]^{T}=[0,0]^{T}\right\}$. On the basis of the invariant manifold, the PMSM system (2) is transformed into

$$
\begin{aligned}
& \dot{\varepsilon}_{1}=-\frac{R}{L} \varepsilon_{1}-\frac{1}{L} n_{p} \psi_{f} \varepsilon_{2}+\frac{1}{L}\left(u_{q}-\pi_{u_{q}}\right), \\
& \dot{\varepsilon}_{2}=\frac{3 n_{p} \psi_{f}}{2 J} \varepsilon_{1}-\frac{B}{J} \varepsilon_{2} .
\end{aligned}
$$

From (10), it is seen that the unmatched disturbance in (2) is lumped into the matched disturbance $\pi_{u_{q}}$, which needs to be compensated.

Denote $\varepsilon_{3}=\pi_{u_{q}}$ as an extended state. Then an augmented system is

$$
\begin{aligned}
& \dot{\varepsilon}_{1}=-\frac{R}{L} \mathcal{E}_{1}-\frac{1}{L} n_{p} \psi_{f} \mathcal{E}_{2}+\frac{1}{L} u_{q}-\frac{1}{L} \mathcal{E}_{3}, \\
& \dot{\varepsilon}_{2}=\frac{3 n_{p} \psi_{f}}{2 J} \varepsilon_{1}-\frac{B}{J} \varepsilon_{2}, \\
& \dot{\varepsilon}_{3}=\varsigma, \\
& e=\varepsilon_{2},
\end{aligned}
$$

where $\varsigma=\dot{\pi}_{u_{q}}$

Assumption 1. The lumped disturbance $\pi_{u_{q}}$ in system (10) and its derivative $\dot{\pi}_{u_{q}}$ are assumed to be bounded and satisfy the condition that $\lim _{t \longrightarrow \infty} \dot{\pi}_{u_{q}}=0$.
An extended state observer for augmented system (11) is designed as

$$
\begin{aligned}
& \dot{\widehat{\mathcal{\varepsilon}}}_{1}=-\frac{R}{L} \widehat{\mathcal{\varepsilon}}_{1}-\frac{1}{L} n_{p} \psi_{f} \widehat{\mathcal{\varepsilon}}_{2}+\frac{1}{L} u_{q}-\frac{1}{L} \widehat{\mathcal{E}}_{3}+l_{1}(e-\widehat{e}), \\
& \dot{\widehat{\mathcal{\varepsilon}}}_{2}=\frac{3 n_{p} \psi_{f}}{2 J} \widehat{\mathcal{E}}_{1}-\frac{B}{J} \widehat{\mathcal{E}}_{2}+l_{2}(e-\widehat{e}), \\
& \dot{\widehat{\mathcal{\varepsilon}}}_{3}=l_{3}(e-\widehat{e}), \\
& \widehat{e}=\widehat{\mathcal{E}}_{2},
\end{aligned}
$$

where $l_{1}, l_{2}, l_{3}$ are observer gains to be determined and $\widehat{\varepsilon}_{1}, \widehat{\varepsilon}_{2}, \widehat{\varepsilon}_{3}$ are estimations of $\varepsilon_{1}, \varepsilon_{2}, \varepsilon_{3}$ in (11). Introducing the estimations, the sliding manifold is designed as

$$
s=\widehat{\varepsilon}_{1}+\beta \widehat{\varepsilon}_{2},
$$

where $\beta>0$ is the parameter to be determined. The outputfeedback sliding mode controller is constructed as

$$
\begin{aligned}
u_{q}= & L\left[\left(\frac{R}{L}-\frac{3 n_{p} \psi_{f} \beta}{2 J}\right) \widehat{\varepsilon}_{1}+\left(\frac{n_{p} \psi_{f}}{L}+\frac{B \beta}{J}\right) \widehat{\varepsilon}_{2}+\frac{1}{L} \widehat{\varepsilon}_{3}\right. \\
& \left.-k_{1} \operatorname{sgn}(s)-k_{2} s\right],
\end{aligned}
$$

where $k_{1}=\rho+\left|\left(l_{1}+\beta l_{2}\right)\left(\varepsilon_{2}-\widehat{\varepsilon}_{2}\right)\right|, k_{2}>0$, and $\rho>0$.

3.2. Stability Analysis. To begin with, we give a lemma as follows, which will play an essential role in analysis.

Lemma 1 (see [39]). If the matrix $A \in \mathbb{R}^{n \times n}$ is Hurwitz, then there is a scalar $c>0$ such that $\left\|e^{A t}\right\| \leq c e^{\left(\lambda_{\max }(A) / 2\right) t}$, where $\lambda_{\max }(A)=\max \left\{\operatorname{Re}\left(\lambda_{i}(A)\right)\right\}$. 
Taking the time derivative of the designed sliding manifold $s$ along (13) and substituting the extended state observer (12) and the output-feedback controller (14), we have

$$
\begin{aligned}
& \dot{\mathcal{s}}=\dot{\widehat{\mathcal{\varepsilon}}}_{1}+\beta \dot{\widehat{\mathcal{\varepsilon}}}_{2} \\
& =-\frac{R}{L} \widehat{\mathcal{E}}_{1}-\frac{1}{L} n_{p} \psi_{f} \widehat{\mathcal{E}}_{2}-\frac{1}{L} \widehat{\mathcal{E}}_{3}+\frac{1}{L} u_{q} \\
& +l_{1}(e-\widehat{e})+\beta\left(\frac{3 n_{p} \psi_{f}}{2 J} \widehat{\mathcal{\varepsilon}}_{1}-\frac{B}{J} \widehat{\varepsilon}_{2}+l_{2}(e-\widehat{e})\right) \\
& =-k_{1} \operatorname{sgn}(s)-k_{2} s+\left(\beta l_{2}+l_{1}\right)(e-\widehat{e}) .
\end{aligned}
$$

Define the candidate Lyapunov function as $V=(1 / 2) s^{2}$. Taking the derivative of $V$ and considering (15), one obtains

$$
\begin{aligned}
\dot{V} & =\dot{s} \leq-k_{1}|s|-k_{2} s^{2}+|s|\left|\left(\beta l_{2}+l_{1}\right)(e-\widehat{e})\right| \\
& <-\rho|s|-k_{2} s^{2}=-\sqrt{2} \rho V^{1 / 2}-2 k_{2} V .
\end{aligned}
$$

As a result, the system trajectories will reach the sliding manifold in a finite time $T_{r}$ with [18]

$$
T_{r} \leq \frac{1}{k_{2}} \ln \left(1+\frac{\sqrt{2} k_{2}}{\rho} V^{1 / 2}(s(0))\right) .
$$

Denote the estimation errors as $\widetilde{e}_{1}=\varepsilon_{1}-\widehat{\varepsilon}_{1}$ and $\widetilde{e}_{2}=\varepsilon_{2}-\widehat{\varepsilon}_{2}$. On sliding motion, there is

$$
s=\widehat{\varepsilon}_{1}+\beta \widehat{\varepsilon}_{2}=0 \Longrightarrow \widehat{\varepsilon}_{1}=-\beta \widehat{\varepsilon}_{2} .
$$

Then, we have

$$
\varepsilon_{1}=\widetilde{e}_{1}+\widehat{\varepsilon}_{1}=\widetilde{e}_{1}-\beta \widehat{\varepsilon}_{2}=\widetilde{e}_{1}-\beta\left(\varepsilon_{2}-\widetilde{e}_{2}\right) .
$$

Define $\xi=\left[\varepsilon_{2}, \tilde{e}_{1}, \tilde{e}_{2}, \tilde{e}_{3}\right]^{T}$. On sliding motion, the reduced-order closed-loop system can be written in a compact form, which is shown as

$$
\dot{\xi}=\Theta \xi+\Psi \varsigma,
$$

where $\Psi=[0,0,0,1]^{T}$, and

$$
\Theta=\left[\begin{array}{cccc}
-\left(\frac{B}{J}+\frac{3 n_{p} \psi_{f} \beta}{2 J}\right) & \frac{3 n_{p} \psi_{f}}{2 J} & \frac{3 n_{p} \psi_{f} \beta}{2 J} & 0 \\
0 & -\frac{R}{L} & -\left(\frac{n_{p} \varphi_{f}}{L}+l_{1}\right) & -\frac{1}{L} \\
0 & \frac{3 n_{p} \psi_{f} \beta}{2 J} & -\left(\frac{B}{J}+l_{2}\right) & 0 \\
0 & 0 & -l_{3} & 0
\end{array}\right] .
$$

The main theorem of this paper is summarized as follows.
Theorem 1. Under Assumption 1, if the observer gains $l_{1}, l_{2}$, and $l_{3}$ in (12) are selected such that the matrix,

$$
\mathbf{M}=\left[\begin{array}{ccc}
-\frac{R}{L} & -\left(\frac{n_{p} \varphi_{f}}{L}+l_{1}\right) & -\frac{1}{L} \\
\frac{3 n_{p} \psi_{f}}{2 J} & -\left(\frac{B}{J}+l_{2}\right) & 0 \\
0 & -l_{3} & 0
\end{array}\right],
$$

is Hurwitz and the controller parameter $\beta$ in (13) and (14) is chosen such that $-\left((B / J)+\left(\left(3 n_{p} \psi_{f} \beta\right) / 2 J\right)\right)<0$, then the estimation errors and the output tracking error of the PMSM system (2) will converge to a bounded neighbourhood of the origin, and the ultimate bound can be made arbitrarily small. Furthermore, if $\dot{\pi}_{u_{q}}$ tends to zero as $t \longrightarrow \infty$, then the closedloop system (20) is globally asymptotically stable.

For better understanding of the main design idea of the proposed control strategy, the detailed proof for Theorem 1 is given in the Appendix. Figure 2 shows the schematic diagram of the proposed output-feedback SMC strategy in implementation.

Remark 1. In parameter tuning, there are usually a number of conflicts/constraints, such as tracking versus disturbance rejection and nominal performance versus robustness. There are three parameters $l_{1}, l_{2}$, and $l_{3}$ to be determined in the extended state observer given in (12). For tuning the observer gains, we usually choose the observer poles $\omega_{o}$ and calculated the values of $l_{1}, l_{2}$, and $l_{3}$ based on the observer expressions (12). If the observer poles are larger, the settling time will be shorter, but the overshoot will be larger, and the measurement noise will be amplified more seriously. In the controller given in (13) and (14), there are three parameters to be assigned, which are $\beta, k_{1}$, and $k_{2}$. The detailed expression of $k_{1}$ is given by $k_{1}=\rho+\left|\left(l_{1}+\beta l_{2}\right)\left(\varepsilon_{2}-\widehat{\varepsilon}_{2}\right)\right|$, and $\rho$ should be positive. The larger $\rho$ means that system states will reach the sliding manifold in a shorter time, but the chattering will be more seriously. The parameters of $\beta$ and $k_{2}$ should be positive. Large values of control gains will reduce the settling time, but the overshoot and the control efforts will be large. Generally, when choosing the parameters, one needs to balance the overshoot, the settling time, and the control efforts.

\section{Simulations}

Simulations, using MATLAB/Simulink, are carried out to validate the control performance of the proposed outputfeedback sliding mode control method, especially in rejecting unmatched disturbances. Table 1 lists the nominal values of the PMSM parameters.

In simulations, to demonstrate the superiority of the proposed output-feedback sliding mode controller, the traditional output-feedback SMC given by (6)-(8) is compared as benchmark method. The observer poles of the proposed method and the traditional method are chosen as 


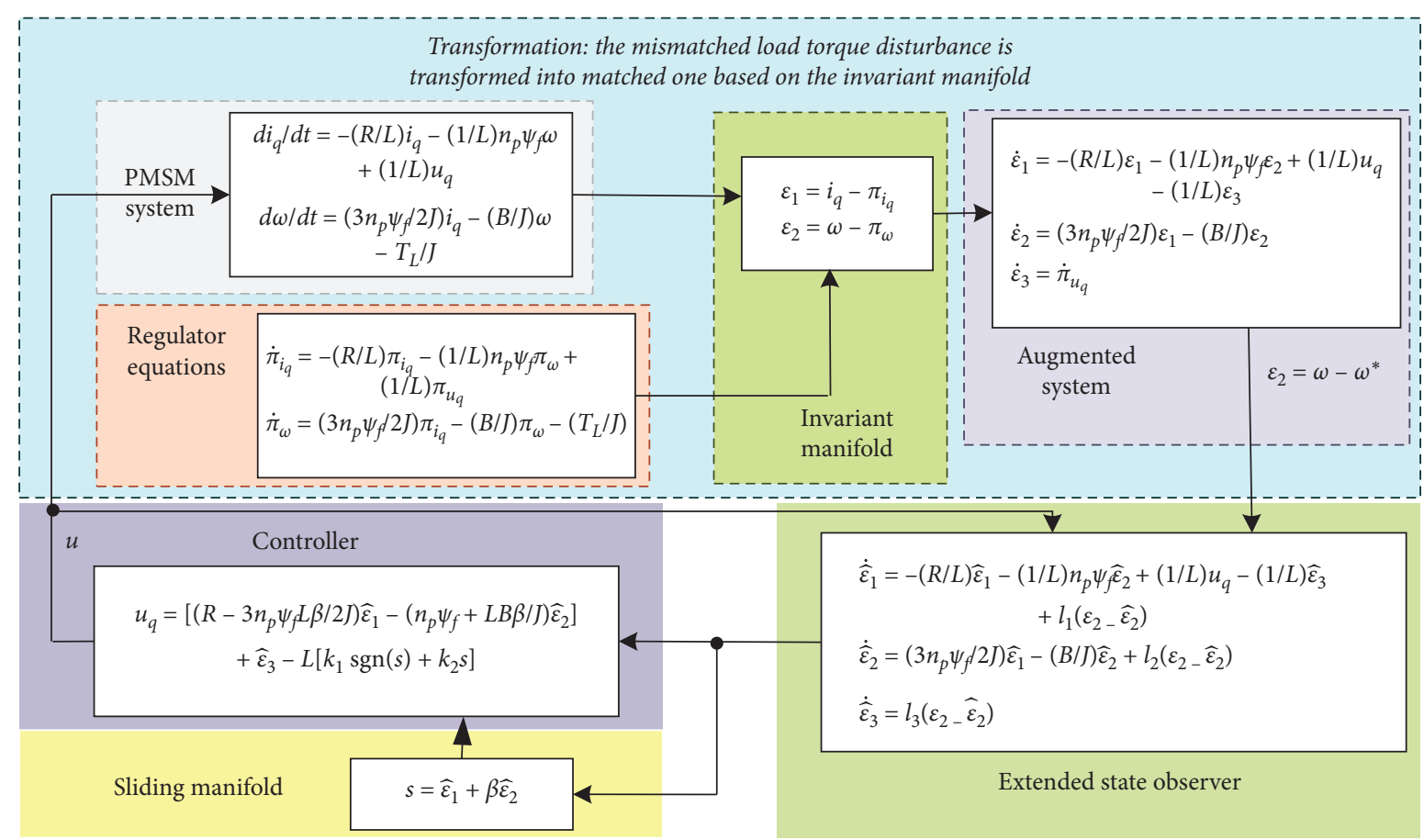

FIGURE 2: Schematic diagram of the proposed output-feedback SMC strategy for PMSM system.

TABle 1: Parameters of PMSM system in simulations.

\begin{tabular}{lcc}
\hline Meanings & Parameters & Nominal value \\
\hline Pole pairs & $n_{p}$ & 4 \\
Rotor flux linkage & $\varphi_{f}$ & $0.402 \mathrm{wb}$ \\
Stator inductance & $L$ & $4 \mathrm{mH}$ \\
Rotor inertia & $J$ & $1.78 \times 10^{-4} \mathrm{~kg} \cdot \mathrm{m}^{2}$ \\
Viscous friction coefficient & $B$ & $7.4 \times 10^{-5} \mathrm{~N} \cdot \mathrm{m} \cdot \mathrm{s} / \mathrm{rad}$ \\
Stator resistance & $R$ & $1.74 \Omega$ \\
\hline
\end{tabular}

$\omega_{o}=-800$ and $\omega_{o}=-1000$, respectively (the reason why we choose different poles for the two observers will be discussed in the following Case 1). The parameters of the two controllers are shown in Table 2.

In this section, we will present three simulation scenarios, which are listed in Table 3.

Case 1. Constant Reference Speed Tracking. In this case, the reference angular velocity is set as $\omega^{*}=150 \mathrm{rad} / s$. The response curves of the angular velocity $\omega$, the $q$-axis current $i_{q}$, and the $q$-axis stator voltage $u_{q}$ are shown in Figure 3 . It is observed that larger observer poles are needed in the traditional output-feedback SMC method to achieve similar control performance with the output-feedback sliding mode controller proposed in this paper. This is the main reason why we choose different poles for the two observers. Moreover, the changes of the switching gains in the two controllers are shown in Figure 4, from which it can be seen that the switching gain $k_{1}$ in the proposed controller is much smaller than that in the traditional one during the transient period.
TABLE 2: Control parameters of two methods.

\begin{tabular}{lcccc}
\hline \multirow{2}{*}{ Methods } & \multicolumn{2}{c}{$\begin{array}{c}\text { Controller } \\
\text { parameters }\end{array}$} & \multicolumn{2}{c}{ Observer parameters } \\
& Symbols & Values & Symbols & Values \\
\hline Proposed & $\beta$ & 1 & $l_{1}$ & 7116.5 \\
Output-feedback & $\rho$ & 1 & $l_{2}$ & 1964.6 \\
SMC & $k_{2}$ & 5 & $l_{3}$ & -151.1376 \\
Traditional & $\beta$ & 80 & $l_{1}$ & 3000 \\
Output-feedback & $\rho$ & 1 & $l_{2}$ & $3 \times 10^{6}$ \\
SMC & $k_{2}$ & 5 & $l_{3}$ & $1 \times 10^{9}$ \\
\hline
\end{tabular}

TABLE 3: Simulation scenarios.

\begin{tabular}{|c|c|c|}
\hline Cases & Scenarios & Details \\
\hline I & $\begin{array}{l}\text { Constant reference } \\
\text { Speed tracking }\end{array}$ & $\omega^{*}=150 \mathrm{rad} / \mathrm{s}$ \\
\hline II & $\begin{array}{l}\text { Sudden load } \\
\text { Torque disturbance }\end{array}$ & $\begin{array}{c}\omega^{*}=150 \mathrm{rad} / \mathrm{s} \\
T_{L}=0 \longrightarrow 1 \mathrm{~N} \cdot \mathrm{m}\end{array}$ \\
\hline III & $\begin{array}{l}\text { Time-varying load } \\
\text { Torque disturbance }\end{array}$ & $\begin{array}{c}\omega^{*}=150 \mathrm{rad} / s \\
T_{L}=0 \stackrel{\sin (2 \pi t) N \cdot m}{\longrightarrow} 2+\sin (2 \pi)\end{array}$ \\
\hline
\end{tabular}

Case 2. Sudden Load Torque Disturbance. When unknown load torques are suddenly imposed, the simulation results are shown by Figure 5. It can be observed from Figure 5(a) that the angular velocity fluctuations of the PMSM are much smaller under the proposed controller than in the traditional method, although the observer poles of the traditional method are larger than those of the proposed one. 


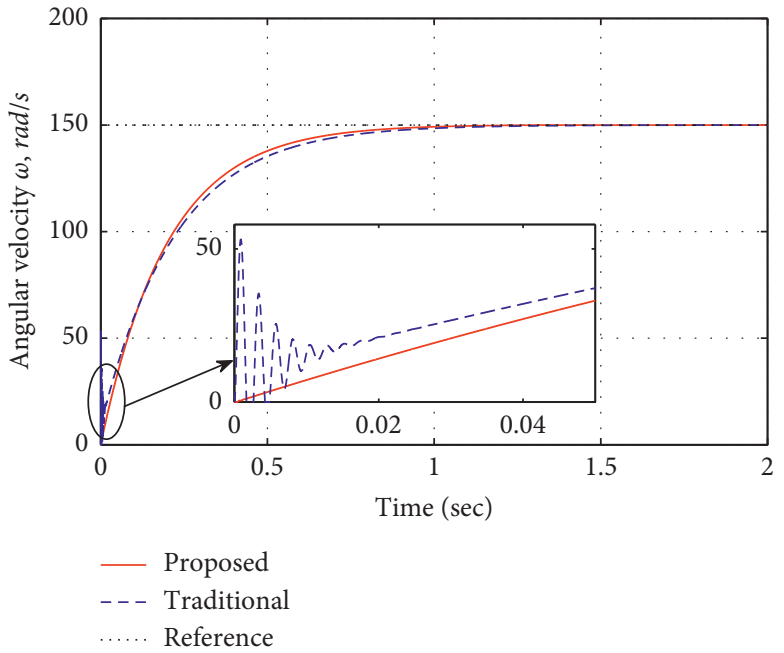

(a)

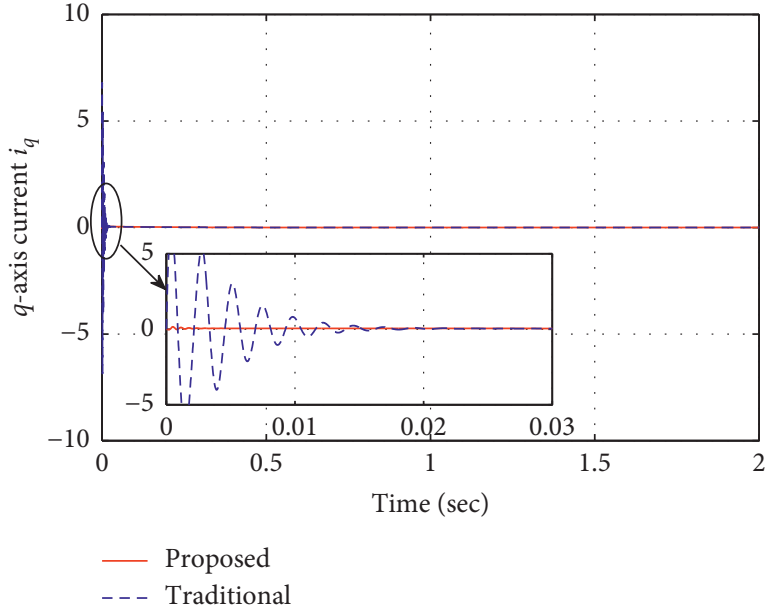

(b)

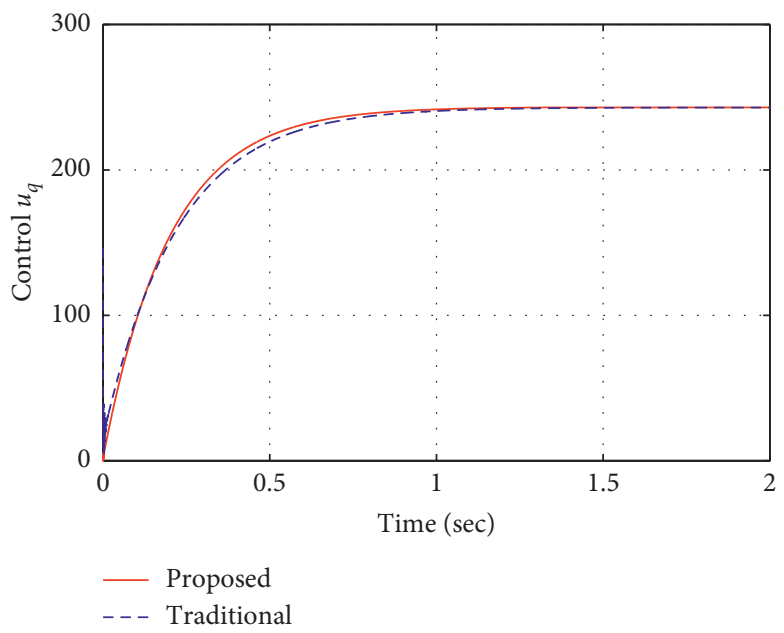

(c)

FIGURE 3: Response curve of the proposed output-feedback SMC and traditional SMC (Case 1): (a) angular velocity $\omega$; (b) $q$-axis current $i_{q}$; (c) control input $u_{q}$.

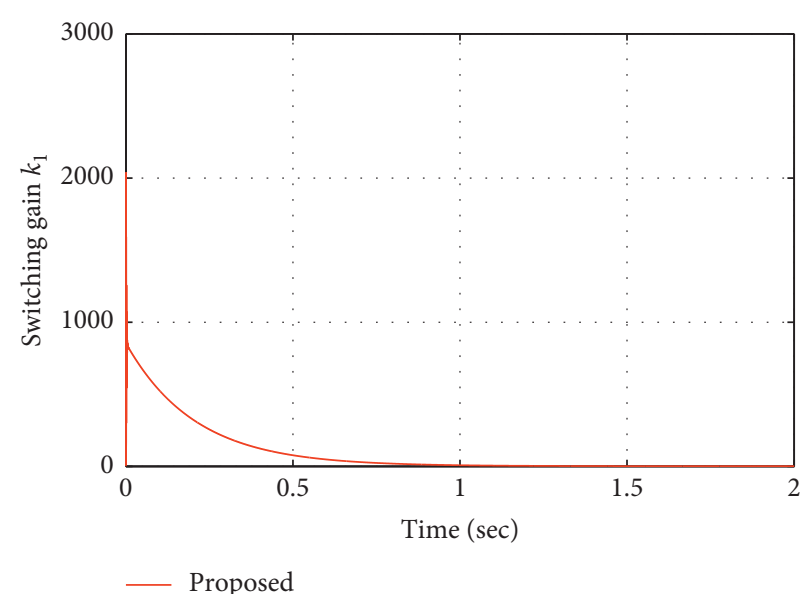

(a)

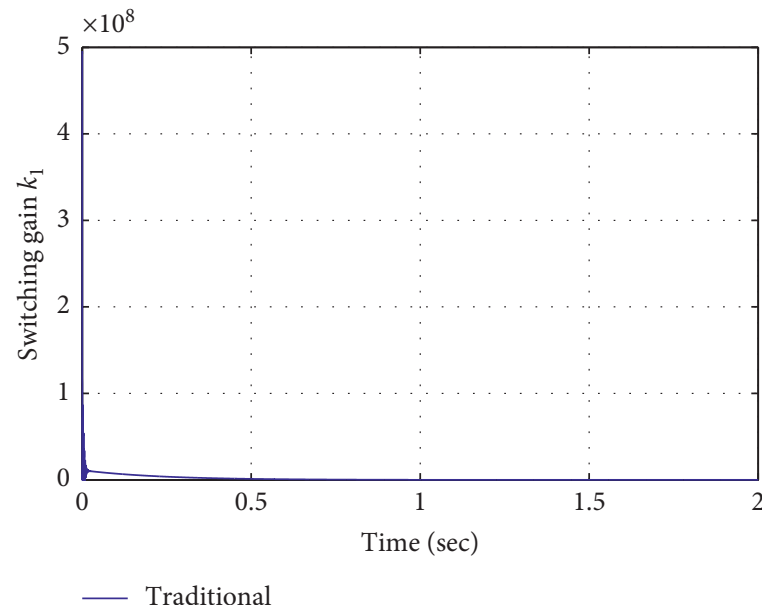

(b)

FIGURE 4: Switching gain of the two controllers: (a) proposed output-feedback SMC; (b) traditional output-feedback SMC. 


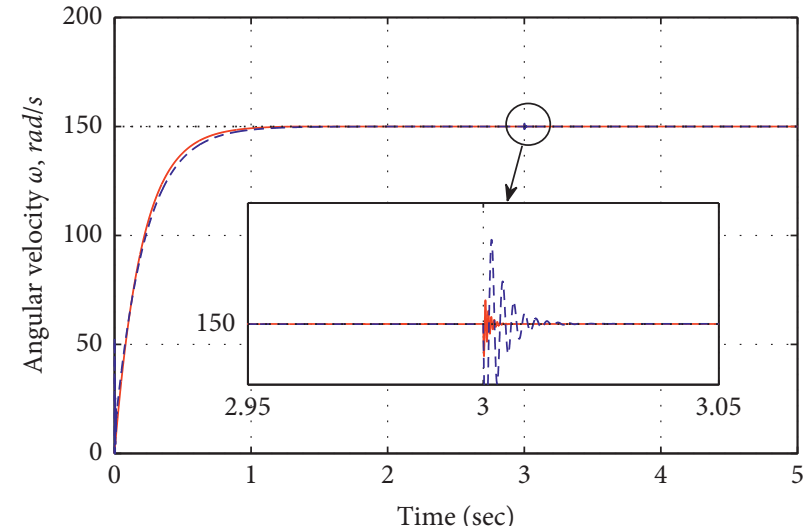

$\begin{array}{ll}\text { _ } & \text { Proposed } \\ \text { - - - Traditional } & \text { Trafionce }\end{array}$

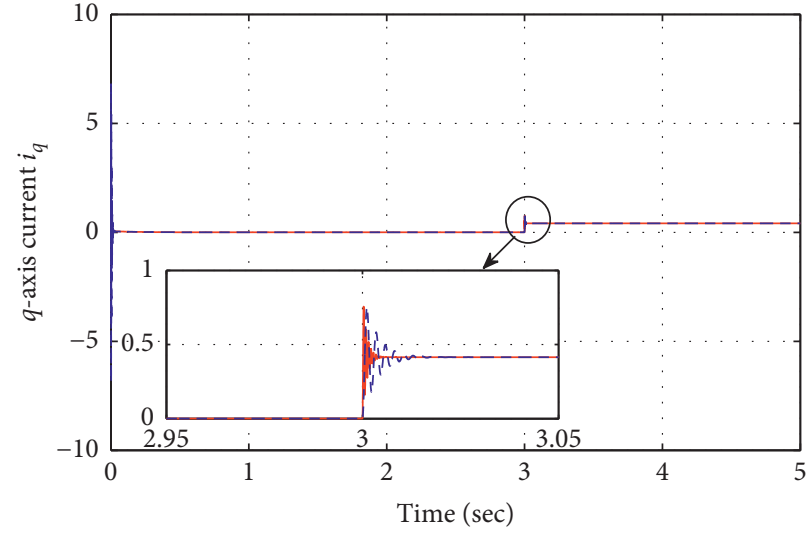

- Proposed

- - - Traditional

(a)

(b)

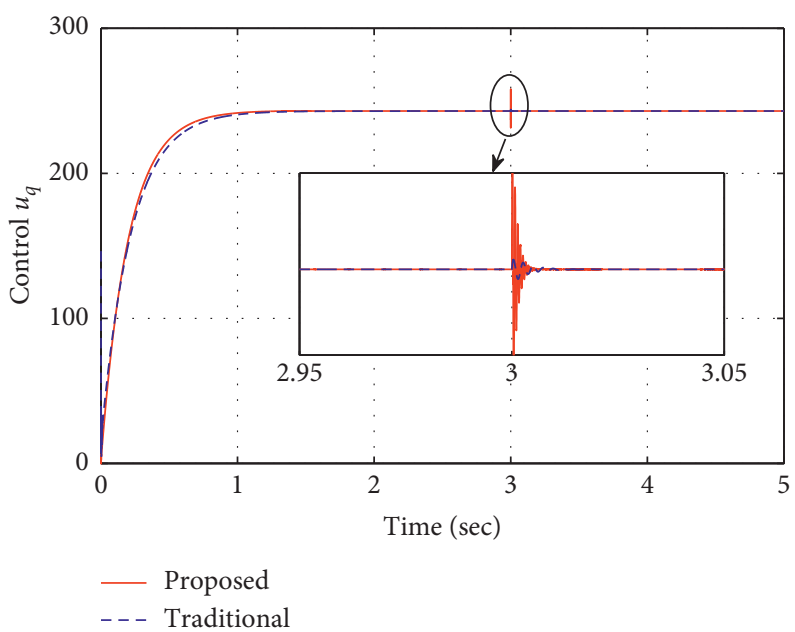

(c)

FIGURE 5: Response curve of the proposed output-feedback SMC and traditional SMC in the presence step load torque disturbance (Case 2): (a) angular velocity $\omega$; (b) $q$-axis current $i_{q}$; (c) control input $u_{q}$.

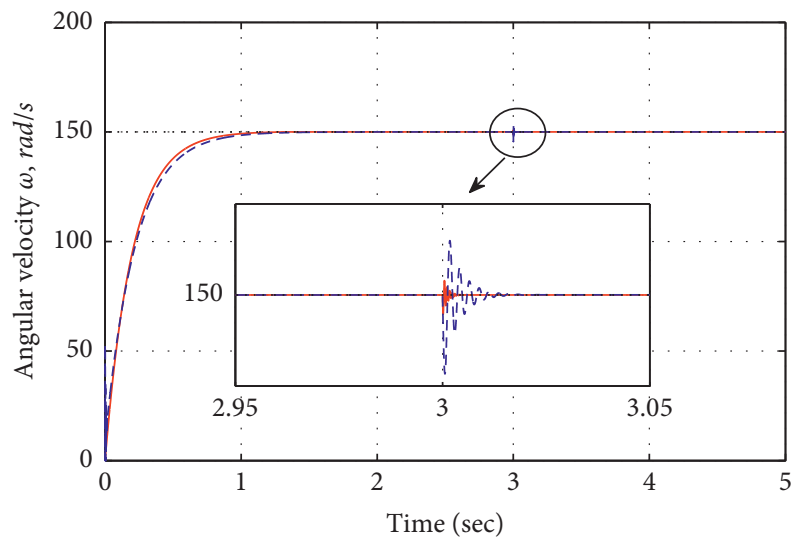

— Proposed

-. - Traditional

...... Reference

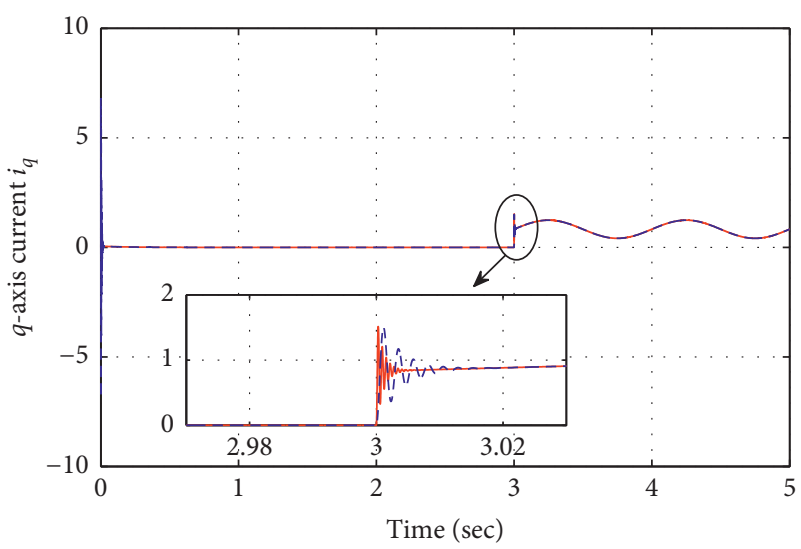

Proposed

- _- Traditional

(a)

FIgure 6: Continued. 


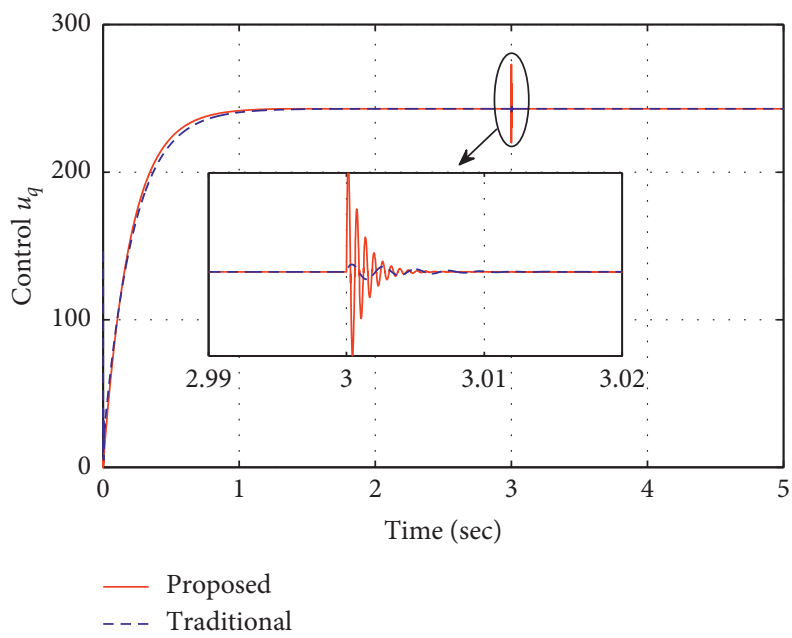

(c)

FIgURE 6: Response curve of the proposed output-feedback SMC and traditional SMC in the presence sinusoidal load torque disturbance (Case 3): (a) angular velocity $\omega$; (b) $q$-axis current $i_{q}$; (c) control input $u_{q}$.

Case 3. Sinusoidal Load Torque Disturbance. Different from Case 3, where constant load torque disturbance is considered; in this case, we aim to evaluate the influences of period time-varying external disturbances on the steady-state performance. Response curves of the angular velocity, the $q$-axis current, and the $q$-axis voltage are shown in Figure 6. It can be seen from Figure 6 that the proposed control method shows better disturbance rejection ability than the traditional output-feedback SMC method.

\section{Conclusion}

This paper has investigated the speed regulation problem for PMSM system subject to unmatched disturbance. Taking advantage of the invariant manifold, the unmatched disturbance has been transformed into matched one and an augmented system has been obtained. An extended state observer has been developed for the augmented system to reconstruct unmeasured states. An output-feedback sliding mode controller, based on invariant manifold, has been proposed to achieve asymptotic tracking. Under the proposed control method, the angular velocity of the PMSM system can track the desired signal asymptotically even in the presence of the unmatched disturbance. Simulations have been carried out to validate the superiority of the controller proposed in this paper.

\section{Appendix}

\section{A. Proof of Theorem 1}

Proof

Step 1. According to the input-to-state stability given in [40], there is a class of $\mathscr{K}$ functions $\alpha$ and a class of $\mathscr{K} \mathscr{L}$ functions $\eta$ such that, for any bounded $\varsigma(t)$ and initial state $\xi(0)$, the solutions of the compact system (20) satisfy $\|\xi(t)\| \leq \eta(\|\xi(0)\|, t)+\alpha\left(\sup _{0 \leq \tau \leq t}|\varsigma(\tau)|\right)$ and $\|\xi(\infty)\| \leq \alpha(\delta)$ $\leq \infty$ with $\left|\pi_{u_{q}}(t)\right| \leq \delta$, where $\delta$ is a positive constant. Therefore, the estimation errors and the output tracking error are bounded.

Step 2. Denote $\widetilde{e}=\left[\widetilde{e}_{1}, \widetilde{e}_{2}, \widetilde{e}_{3}\right]^{T}$. Combining the augmented system (11), the extended state observer (12), and the reduced-order closed-loop system (20), the subsystem of observer estimation error is given as

$$
\dot{\tilde{\mathbf{e}}}=\mathbf{M} \widetilde{\mathbf{e}}+\mathbf{N} \varsigma,
$$

where $\mathbf{N}=[0,0,1]^{T}$. Based on the comparison lemma [40], there is

$$
\widetilde{\mathbf{e}}(t)=e^{\mathbf{M} t} \widetilde{\mathbf{e}}(0)+\int_{0}^{t} e^{\mathbf{M}(t-s)} \mathbf{N} \varsigma(s) \mathrm{d} s
$$

In general, if the observer poles are chosen as $\omega_{o}<0$, then, combining with Lemma 1, we have

$$
\begin{aligned}
\|\widetilde{\mathbf{e}}(t)\| \leq & \int_{0}^{t}\left\|e^{(\overline{\mathbf{A}}-\mathbf{L} \overline{\mathbf{C}})(t-s)}\right\|\|\mathbf{N}\||\varsigma(s)| \mathrm{d} s \\
& +\left\|e^{(\overline{\mathbf{A}}-\mathbf{L} \overline{\mathbf{C}}) t}\right\|\|\widetilde{\mathbf{e}}(0)\| \\
\leq & \delta \int_{0}^{t} c_{2} e^{\left(\omega_{o} / 2\right)(t-s)} \mathrm{d} s+c_{1} e^{\left(\omega_{o} / 2\right) t}\|\widetilde{\mathbf{e}}(0)\| \\
\leq & \frac{2 \delta c_{2}\left(1-e^{\left(\omega_{o} / 2\right) t}\right)}{-\omega_{o}}+c_{1} e^{\left(\omega_{o} / 2\right) t}\|\widetilde{\mathbf{e}}(0)\|,
\end{aligned}
$$

where $c_{1}>0$ and $c_{2}>0$ are constants. As time goes by, there is $\lim _{t \rightarrow \infty}\|\widetilde{\mathbf{e}}(t)\|=\left(2 \delta c_{2} /-\omega_{o}\right)$. If the observer gains in (12) are selected such that the matrix $\mathbf{M}$ is Hurwitz, then the estimation errors are bounded and can be arbitrarily small by increasing observer poles.

It can be obtained from (20) that the state subsystem is 


$$
\dot{\varepsilon}_{2}=-\left(\frac{B}{J}+\frac{3 n_{p} \varphi_{f} \beta}{2 J}\right) \varepsilon_{2}+\frac{3 n_{p} \varphi_{f}}{2 J} \widetilde{e}_{1}+\frac{3 n_{p} \psi_{f} \beta}{2 J} \widetilde{e}_{2}
$$

Based on the comparison lemma given in [40], we have the following inequality:

$$
\begin{aligned}
\varepsilon_{2}(t)= & e^{-\left((B / J)+\left(3 n_{p} \varphi_{f} \beta / 2 J\right)\right) t} \varepsilon_{2}(0) \\
& +\int_{0}^{t} e^{-\left((B / J)+\left(3 n_{p} \varphi_{f} \beta / 2 J\right)\right)(t-s)}\left(\frac{3 n_{p} \varphi_{f}}{2 J} \widetilde{e}_{1}(\tau)\right. \\
& \left.+\frac{3 n_{p} \psi_{f} \beta}{2 J} \widetilde{e}_{2}(\tau)\right) \mathrm{d} \tau .
\end{aligned}
$$

Denote a positive constant

$$
\hbar=\left|\frac{3 n_{p} \varphi_{f}}{2 J} \widetilde{e}_{1}(t)+\frac{3 n_{p} \psi_{f} \beta}{2 J} \widetilde{e}_{2}(t)\right| .
$$

Since the parameter $\beta$ is chosen such that $-\left((B / J)+\left(3 n_{p} \psi_{f} \beta / 2 J\right)\right)<0$, similar to (A.3), one obtains

$$
\begin{aligned}
\left|\varepsilon_{2}(t)\right| \leq & m_{1} e^{-\left((B / J)+\left(3 n_{p} \psi_{f} \beta / 2 J\right)\right) t}\left|\varepsilon_{2}(0)\right| \\
& +\frac{2 m_{2} \hbar}{(B / J)+\left(3 n_{p} \psi_{f} \beta / 2 J\right)} \\
& \cdot\left(1-e^{-\left(\left((B / J)+\left(3 n_{p} \psi_{f} \beta / 2 J\right)\right) / 2\right) t}\right),
\end{aligned}
$$

where $m_{1}, m_{2}>0$. As a result, we have

$$
\lim _{t \rightarrow \infty} \varepsilon_{2}(t)=\frac{2 m_{2} \hbar}{\left((B / J)+\left(3 n_{p} \psi_{f} \beta / 2 J\right)\right)} .
$$

It is noted that the ultimate bound of estimation errors can be made arbitrarily small; therefore, the ultimate bound of the tracking error $\varepsilon_{2}(t)$ can be arbitrarily small.

Step 3. Furthermore, if the assumption that $\lim _{t \rightarrow \infty} \dot{\pi}_{u_{q}}=0$ is satisfied, following the method given in [41], one obtains

$$
\begin{aligned}
\lim _{t \longrightarrow \infty} \widetilde{\mathbf{e}}(t) & =\lim _{s \longrightarrow 0} \widetilde{\mathbf{E}}(s)=\lim _{s \longrightarrow 0}[s \mathbf{I}-\mathbf{M}]^{-1} \mathbf{N} \mathscr{L}(\varsigma(t)) \\
& =\lim _{s \longrightarrow 0}[s \mathbf{I}-\mathbf{M}]^{-1} \mathbf{N} \cdot \lim _{t \longrightarrow \infty} \varsigma(t)=\mathbf{0} .
\end{aligned}
$$

Based on the above analysis, we have

$$
\begin{aligned}
\lim _{t \longrightarrow \infty} \varepsilon_{2}(t)= & \lim _{s \longrightarrow 0}\left(s+\left(\frac{B}{J}+\frac{3 n_{p} \psi_{f} \beta}{2 J}\right)\right)^{-1} \\
& \cdot \mathscr{L}\left(\frac{\left.3 n_{p} \psi_{f_{\widetilde{e}_{1}}}(t)+\frac{\left.3 n_{p} \psi_{f} \beta_{\widetilde{e}_{2}}(t)\right)}{2 J}\right)}{=} \lim _{s \longrightarrow 0}\left(s+\left(\frac{B}{J}+\frac{3 n_{p} \psi_{f} \beta}{2 J}\right)\right)^{-1}\right. \\
& \cdot \lim _{t \longrightarrow \infty}\left(\frac{3 n_{p} \psi_{f}}{2 J} \widetilde{e}_{1}(t)+\frac{3 n_{p} \psi_{f} \beta}{2 J} \widetilde{e}_{2}(t)\right) .
\end{aligned}
$$

Taking (19) in mind, one obtains $\lim _{t \longrightarrow \infty} \varepsilon_{1}(t)=\lim _{t \longrightarrow \infty} \tilde{e}_{1}-\beta \widetilde{\varepsilon}_{2}=\widetilde{e}_{1}-\beta\left(\varepsilon_{2}-\widetilde{e}_{2}\right)=0$.

Since $\varepsilon_{2}=\omega-\omega^{*}$, we have $\lim _{t \rightarrow \infty} \omega=\omega^{*}$. As a result, the angular velocity of the PMSM system can track the desired reference signal asymptotically. This completes the proof.

\section{Data Availability}

The mathematical model of PMSM system data used to support the findings of this study have been deposited in the Web of Science repository (DOI: 10.1109/TIE.2009.2024655).

\section{Conflicts of Interest}

The authors declare that they have no conflicts of interest.

\section{Acknowledgments}

This work was supported in part by the National Natural Science Foundation of China under Grant 61973080.

\section{References}

[1] P. Krause, O. Wasynczuk, S. Sudhoff, and S. Pekarek, Analysis of Electric Machinery and Drive Systems, Wiley, Hoboken, NJ, USA, 2013.

[2] S. Li and Z. Liu, "Adaptive speed control for permanentmagnet synchronous motor system with variations of load inertia," IEEE Transactions on Industrial Electronics, vol. 56, no. 8, pp. 3050-3059, 2009.

[3] R. He and Q. Han, "Dynamics and stability of permanentmagnet synchronous motor," Mathematical Problems in Engineering, vol. 2017, Article ID 4923987, 8 pages, 2017.

[4] W.-C. Gan and L. Qiu, "Torque and velocity ripple elimination of AC permanent magnet motor control systems using the internal model principle," IEEE/ASME Transactions on Mechatronics, vol. 9, no. 2, pp. 436-447, 2004.

[5] G. J. Wang, C. T. Fong, and K. J. Chang, "Neural-networkbased self-tuning PI controller for precise motion control of PMAC motors," IEEE Transactions on Industrial Electronics, vol. 48, no. 2, pp. 408-415, 2001.

[6] A. V. Sant and K. R. Rajagopal, "PM synchronous motor speed control using hybrid fuzzy-PI with novel switching functions," IEEE Transactions on Magnetics, vol. 45, no. 10, pp. 4672-4675, 2009.

[7] T.-B. T. Nguyen, T.-L. Liao, H.-H. Kuo, and J.-J. Yan, “An improved adaptive tracking controller of permanent magnet synchronous motor," Abstract and Applied Analysis, vol. 2014, Article ID 987308, 12 pages, 2014.

[8] Y. Yan, J. Yang, Z. Sun, C. Zhang, S. Li, and H. Yu, "Robust speed regulation for PMSM servo system with multiple sources of disturbances via an augmented disturbance observer," IEEE/ASME Transactions on Mechatronics, vol. 23, no. 2, pp. 769-780, 2018.

[9] F. F. M. El-Sousy, "Hybrid $\$\{\backslash \mathrm{rm} \mathrm{H}\}\{\backslash$ infty\} $\$$-Based waveletneural-network tracking control for permanent-magnet synchronous motor servo drives," IEEE Transactions on Industrial Electronics, vol. 57, no. 9, pp. 3157-3166, 2010.

[10] H. A. Zarchi, G. R. A. Markadeh, and J. Soltani, "Direct torque and flux regulation of synchronous reluctance motor drives based on input-output feedback linearization," Energy Conversion and Management, vol. 51, no. 1, pp. 71-80, 2010. 
[11] Y.-H. Lan and L. Lei-Zhou, "Backstepping control with disturbance observer for permanent magnet synchronous motor," Journal of Control Science and Engineering, vol. 2018, Article ID 4938389, 8 pages, 2018.

[12] R. J. Wai, "Hybrid fuzzy neural-network control for nonlinear motor toggle servomechanism," IEEE Transactions on Control Systems Technology, vol. 10, no. 4, pp. 519-532, 2002.

[13] S. Li, H. Liu, and S. Ding, "A speed control for a PMSM using finite-time feedback control and disturbance compensation," Transactions of the Institute of Measurement and Control, vol. 32, no. 2, pp. 170-187, 2010.

[14] K. Mei, L. Ma, R. He, and S. Ding, "Finite-time controller design of multiple integrator nonlinear systems with input saturation," Applied Mathematics and Computation, vol. 372, Article ID 124986, 2020.

[15] Y. Luo, Y. Chen, H.-S. Ahn, and Y. Pi, "Fractional order robust control for cogging effect compensation in PMSM position servo systems: stability analysis and experiments," Control Engineering Practice, vol. 18, no. 9, pp. 1022-1036, 2010.

[16] L. Liu, W. X. Zheng, and S. Ding, "An adaptive SOSM controller design by using a sliding-mode-based filter and its application to buck converter," IEEE Transactions on Circuits and Systems I: Regular Papers, vol. 67, no. 7, pp. 2409-2418, 2020.

[17] C.-F. Huang, J.-S. Lin, T.-L. Liao, C.-Y. Chen, and J.-J. Yan, "Quasi-sliding mode control of chaos in permanent magnet synchronous motor," Mathematical Problems in Engineering, vol. 2011, Article ID 964240, 10 pages, 2011.

[18] S. Yu, X. Yu, B. Shirinzadeh, and Z. Man, "Continuous finitetime control for robotic manipulators with terminal sliding mode," Automatica, vol. 41, no. 11, pp. 1957-1964, 2005.

[19] J. Yuan, S. Ding, and K. Mei, "Fixed-time SOSM controller design with output constraint," Nonlinear Dynamics, vol. 102, no. 3, pp. 1567-1583, 2020.

[20] V. Utkin, "Variable structure systems with sliding modes," IEEE Transactions on Automatic Control, vol. 22, no. 2, pp. 212-222, 1977.

[21] V. Utkin, A. Poznyak, Y. Orlov, and A. Polyakov, "Conventional and high order sliding mode control," Journal of the Franklin Institute, vol. 357, no. 15, pp. 10244-10261.

[22] V. Utkin, Sliding Modes in Control Optimisation, SpringerVerlag, Berlin, Germany, 1992.

[23] A. Levant, "Higher-order sliding modes, differentiation and output-feedback control," International Journal of Control, vol. 76, pp. 9-10, 2003.

[24] J. Yang, S. Li, and X. Yu, "Sliding-mode control for systems with mismatched uncertainties via a disturbance observer," IEEE Transactions on Industrial Electronics, vol. 60, no. 1, pp. 160-169, 2013.

[25] M. Zhang, F. Xiao, R. Shao, and Z. Deng, "Robust fault detection for permanent-magnet synchronous motor via adaptive sliding-mode observer," Mathematical Problems in Engineering, vol. 2020, Article ID 6405923, 6 pages, 2020.

[26] Y. Feng, X. Yu, and F. Han, "High-order terminal slidingmode observer for parameter estimation of a permanentmagnet synchronous motor," IEEE Transactions on Industrial Electronics, vol. 60, no. 10, pp. 4272-4280, 2013.

[27] P. Xia, Y. Deng, Z. Wang, and H. Li, "Speed adaptive sliding mode control with an extended state observer for permanent magnet synchronous motor," Mathematical Problems in Engineering, vol. 2018, Article ID 6405923, 13 pages, 2018.
[28] J. M. Daly and D. W. L. Wang, "Output feedback sliding mode control in the presence of unknown disturbances," Systems \& Control Letters, vol. 58, no. 3, pp. 188-193, 2009.

[29] M. V. Basin, C. B. Panathula, Y. B. Shtessel, and P. C. R. Ramirez, "Continuous finite-time higher order output regulators for systems with unmatched unbounded disturbances," IEEE Transactions on Industrial Electronics, vol. 63, no. 8, pp. 5036-5043, 2016.

[30] J. P. V. S. Cunha, R. R. Costa, F. Lizarralde, and L. Hsu, "Peaking free variable structure control of uncertain linear systems based on a high-gain observer," Automatica, vol. 45, no. 5, pp. 1156-1164, 2009.

[31] J. Han, "From PID to active disturbance rejection control," IEEE Transactions on Industrial Electronics, vol. 56, no. 3, pp. 900-906, 2009.

[32] Y. Xia, F. Pu, M. Fu, and L. Ye, "Modeling and compound control for unmanned turret system with coupling," IEEE Transactions on Industrial Electronics, vol. 63, no. 9, pp. 5794-5803, 2016.

[33] J. Mao, J. Yang, S. Li, Y. Yan, and Q. Li, “Output feedbackbased sliding mode control for disturbed motion control systems via a higher-order ESO approach," IET Control Theory \& Applications, vol. 12, no. 15, pp. 2118-2126, 2018.

[34] J. A. Moreno, "Discontinuous integral control for mechanical systems," in Proceedings of 14th International Workshop on Variable Structure Systems, pp. 142-147, IEEE, Nanjing, China, June 2016.

[35] L. Zhang, J. Yang, S. Li, and X. Yu, "Invariant manifold based output-feedback sliding mode control for systems with mismatched disturbances," IEEE Transactions on Circuits and Systems II: Express Briefs, vol. 68, no. 3, pp. 933-937.

[36] A. Isidori and C. I. Byrnes, "Output regulation of nonlinear systems," IEEE Transactions on Automatic Control, vol. 35, no. 2, pp. 131-140, 1990.

[37] M. Hosseini-Pishrobat, J. Keighobadi, A. Oveisi, and T. Nestorović, "Robust linear output regulation using extended state observer," Mathematical Problems in Engineering, vol. 2018, Article ID 4095473, 12 pages, 2018.

[38] A. Mesbahi and J. Mohammadpour Velni, "Cooperative output regulation of multiagent linear parameter-varying systems," Mathematical Problems in Engineering, vol. 2017, Article ID 8489308, 10 pages, 2017.

[39] W. Zhang, "Stability of networked control systems," Ph. D. Thesis, Case Western Reserve University, Department of Electrical Engineering and Computer Science, Cleveland, OH, USA, 2001.

[40] H. Khalil, Nonlinear Systems, Prentice-Hall, Upper Saddle River, NJ, USA, 3rd edition, 2002.

[41] S. Li, J. Yang, W.-H. Chen, and X. Chen, "Generalized extended state observer based control for systems with mismatched uncertainties," IEEE Transactions on Industrial Electronics, vol. 59, no. 12, pp. 4792-4802, 2012. 